Gut, 1975, 16, 99-104

\title{
The exsorption characteristics of various sugars
}

\author{
A. T. R. AXON AND B. CREAMER \\ From the Gastrointestinal Laboratory, St Thomas' Hospital, London
}

SUMMARY Various sugars were administered intravenously to rabbits and the rate of exsorption from plasma to intestinal lumen was measured. The exsorption rates of lactulose, mannose, and xylose were directly related to their plasma concentration but varied according to their molecular weight. Glucose and galactose did not appear in the intestinal lumen until very high plasma concentrations were reached. The mucosal barrier which prevented glucose and galactose exsorption was abolished when luminal phlorizin $\mathrm{M} \times 10^{-2}$ or another actively absorbed sugar was placed in the lumen. 2:4 Dinitrophenol $\mathrm{M} \times 10^{-3}$ and the absence of the sodium ion did not impair the efficiency of the barrier.

When the plasma glucose concentration was raised to very high levels, glucose began to appear in the lumen; further increases in the glucose concentration then produced a rapid exsorption of glucose.

An important function of the small bowel mucosa is to act as a barrier which prevents the contents of the body from diffusing into the intestinal lumen, but at the same time it has to behave as a permeable surface allowing substances to pass from the lumen into the circulation. If the efficiency of the barrier is tested in the rabbit, it can be shown that it is only partially effective in that many substances pass out of the plasma and are found in the intestinal lumen. This process is best called exsorption (Code, 1960). Loehry, Axon, Hilton, Hider, and Creamer (1970) investigated the exsorption of water-soluble substances in rabbits and demonstrated a direct relationship between the rate of exsorption and plasma concentration; they also showed that large molecules exsorb more slowly than small ones and that it was possible to predict the exsorption rate of a substance if its molecular weight and plasma concentration were known. Glucose differed from the other substances which they tested; according to its molecular weight it should have had an exsorption rate of $0.3 \mathrm{ml} / \mathrm{min}$, but in fact no exsorption at all was demonstrable at the plasma concentration studied.

The purpose of this paper is to compare the exsorption pattern of a series of sugars and to discuss the findings as they relate to special absorption mechanisms.

Received for publication 4 December 1974.

\section{Methods and Materials}

New Zealand white or Canadian albino rabbits were used; their weights varied between 1.6 and 3.0 $\mathrm{kg}$. Experiments were carried out under nembutal anaesthesia and at the end of each experiment the rabbits were killed with an overdose of nembutal before regaining consciousness.

Following intravenous anaesthesia, an external jugular vein was cannulated with PVC tubing to facilitate intravenous infusions. The carotid artery on the opposite side was similarly cannulated so that blood samples could be easily obtained.

The abdomen was opened by a midline incision and the renal pedicles were tied off to prevent the loss of injected materials by this route. The jejunum was cannulated distal to the ligament of Treitz and the ileum was cannulated proximal to the appendix mesentery. Care was taken to ensure that there was no obstruction to the vascular supply of the bowel.

A variety of solutions at $40^{\circ} \mathrm{C}$ were perfused through the small intestine and allowed to run out of the ileal cannula into a collecting cylinder. After some preliminary perfusions to clear the bowel of faecal debris, the pump was adjusted to deliver saline at a rate of $10 \mathrm{ml} / \mathrm{minute}$. Substances to be tested were injected into the jugular cannula and after an equilibration period of 15 minutes, intestinal perfusate was collected for a timed 10-minute period; at five minutes a blood sample was withdrawn from 
the arterial cannula. The concentrations of test substance in both plasma and perfusate were determined experimentally. In most experiments four sequential injections were made, allowing four corresponding exsorption periods to be studied.

The sugars tested were glucose, galactose, xylose, mannose, and lactulose. Each injection solution contained a test sugar together with $1 \mathrm{~g}$ of creatinine. Creatinine was included as a standard; its plasma concentration and exsorption rate were measured at the same time as that of the test sugar. The creatinine standard was used to reduce experimental variation. Loehry showed that the plasma clearance of creatinine by rabbit intestine is $0.51 \mathrm{ml} / \mathrm{min}$ and this has been taken as the standard. All sugar exsorption rates were standardized to conform to a rabbit with a creatinine clearance of 0.51 . This was done in the following way:

Standardized sugar exsorption $=$

$$
\frac{\text { test sugar exsorption } \times 0.51}{\text { test creatinine clearance }} \text {. }
$$

Sugar concentrations were estimated by quantitative paper chromatography (Menzies, 1973).

Seven different perfusion fluids were used, namely, (1) physiological saline, (2) physiological saline containing $2: 4$ dinitrophenol (DNP) $10^{-3} \mathrm{M}$, (3) physiological saline containing phlorizin $10^{-3} \mathrm{M}$, (4) physiological saline containing phlorizin $10^{-2} \mathrm{M}$, (5) isotonic mannitol, (6) isotonic potassium chloride, and (7) 1:1 physiological saline : isotonic glucose (this gives a glucose concentration of 140 $\mu \mathrm{M} / \mathrm{ml})$.

\section{Results}

Lactulose, mannose, and xylose behaved similarly (figs 1, 2, and 3). There was a direct relationship between exsorption rate and plasma concentration.

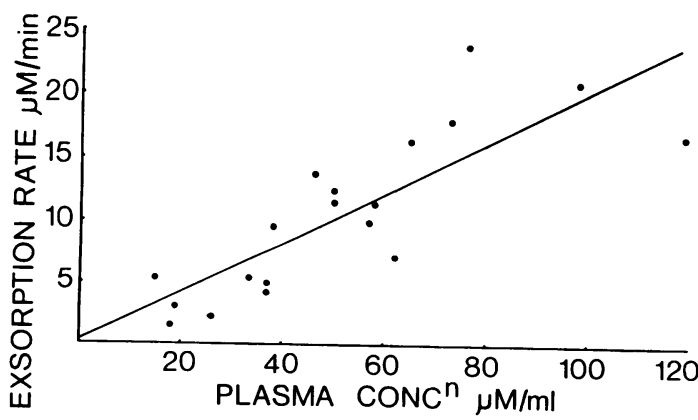

Fig 1 The exsorption pattern of lactulose $(y=0 \cdot 20 x$ $+0 \cdot 25, r=0 \cdot 828$ ).

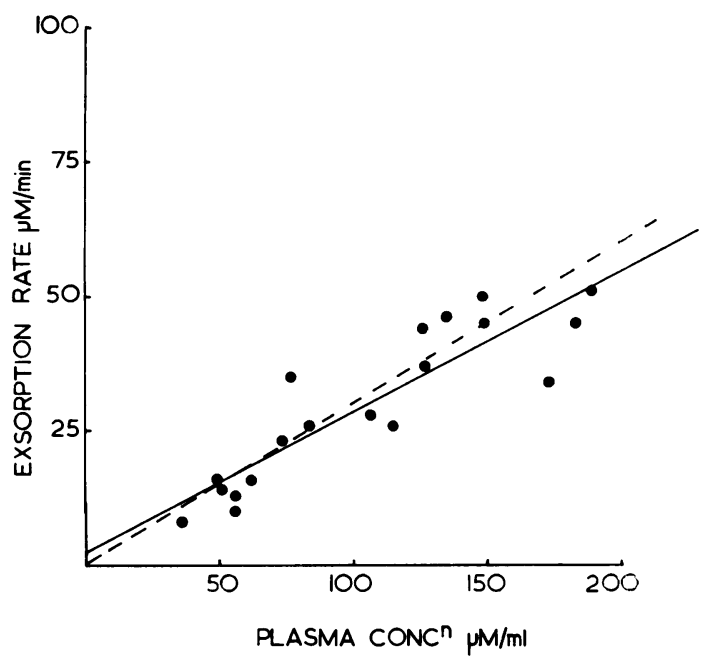

Fig 2 The exsorption pattern of mannose. The regression line is the continuous line $(y=0 \cdot 26 x+$ $2 \cdot 31, r=0 \cdot 817)$. The broken line represents an intestinal clearance of $0.3 \mathrm{ml} / \mathrm{min}$.

The intestinal clearance of the three sugars was $0 \cdot 20$, 0.26 , and $0.37 \mathrm{ml} / \mathrm{min}$ respectively; the clearance of xylose was significantly greater than that of mannose and lactulose $(P<0.01)$. The clearance of lactulose and mannose were not significantly different.

The exsorption patterns of glucose and galactose were quite different. At low concentrations there appeared to be a barrier preventing the sugars from reaching the lumen (figs 4 and 5), virtually no exsorption took place until the plasma concentra-

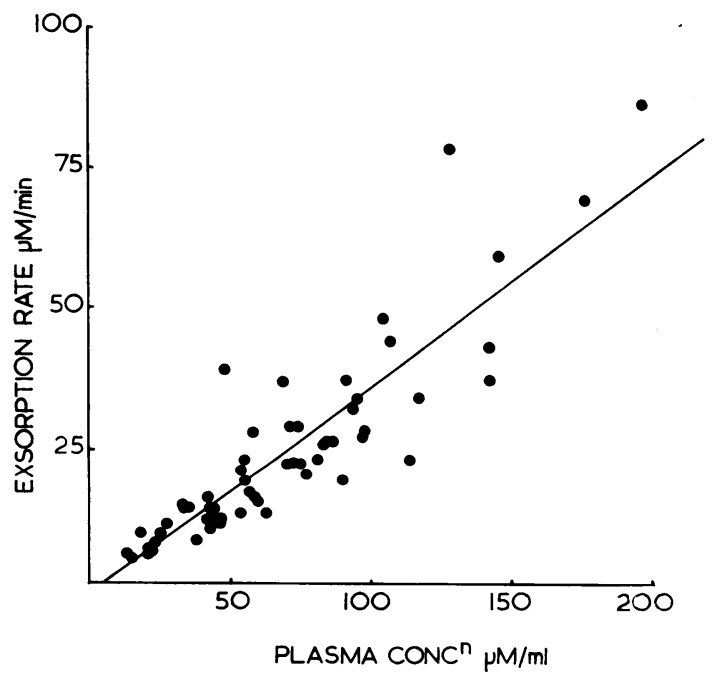

Fig 3 The exsorption pattern of xylose $(y=0 \cdot 37 x-$ $1.53, r=0.881)$. 


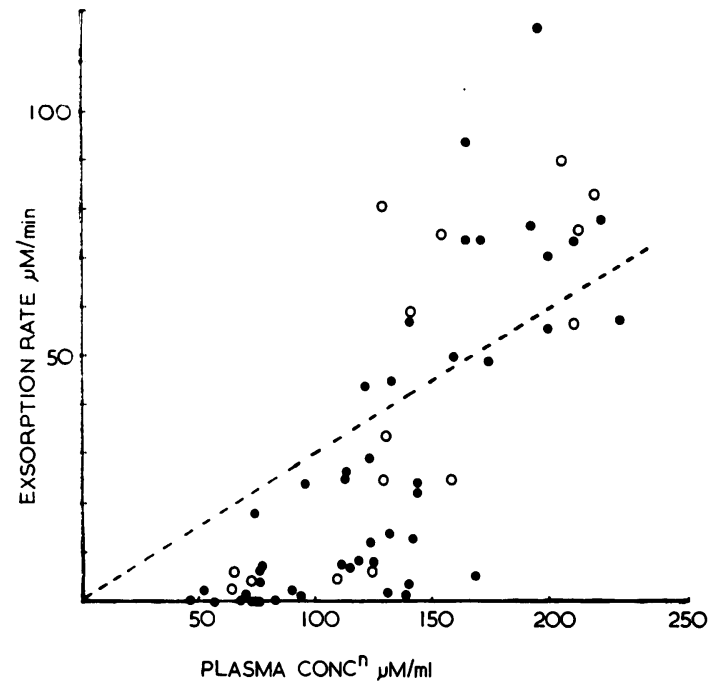

Fig 4 Glucose exsorption. The closed circles represent the exsorption pattern of glucose when the intestinal perfusate comprised physiological saline alone; the open circles show the effect of including DNP in the saline perfusate. The broken line represents an intestinal clearance of $0.3 \mathrm{ml} / \mathrm{min}$.

tion reached $50 \mu \mathrm{M} / \mathrm{ml}$ and even at $100 \mu \mathrm{M} / \mathrm{ml}$ exsorption was slow. When, however, the plasma concentration rose above this, the exsorption rate rapidly increased giving an intestinal clearance of $0.3 \mathrm{ml} / \mathrm{min}$ at about $150 \mu \mathrm{M} / \mathrm{ml}$, while further increases in the plasma glucose concentration produced even higher exsorption rates.

The presence of competitive inhibitors in the lumen (figs 6, 7, and 8) caused a dramatic change in the exsorption pattern of glucose and galactose. Phlorizin $\mathrm{M} \times 10^{-3}$ partially abolished the mucosal barrier to glucose. When the concentration was increased to $\mathrm{M} \times 10^{-2}$, the barrier disappeared completely and the intestinal clearance approached $0.3 \mathrm{ml} / \mathrm{min}$ over the whole range of plasma concentrations and the pattern came to resemble that of mannose, another hexose. Luminal glucose in a concentration of $140 \mu \mathrm{M} / \mathrm{ml}$ had a similar effect on galactose exsorption.

The metabolic inhibitor DNP had no effect on the exsorption pattern of glucose (see fig 4) and the removal of sodium from the luminal perfusate had no effect on galactose exsorption (see fig 5): in both cases the barrier remained intact. The concentration of sugar in the perfusate during these experiments varied between 0 and $12 \mu \mathrm{M} / \mathrm{ml}$.

\section{Discussion}

The exact route taken by exsorbing molecules is

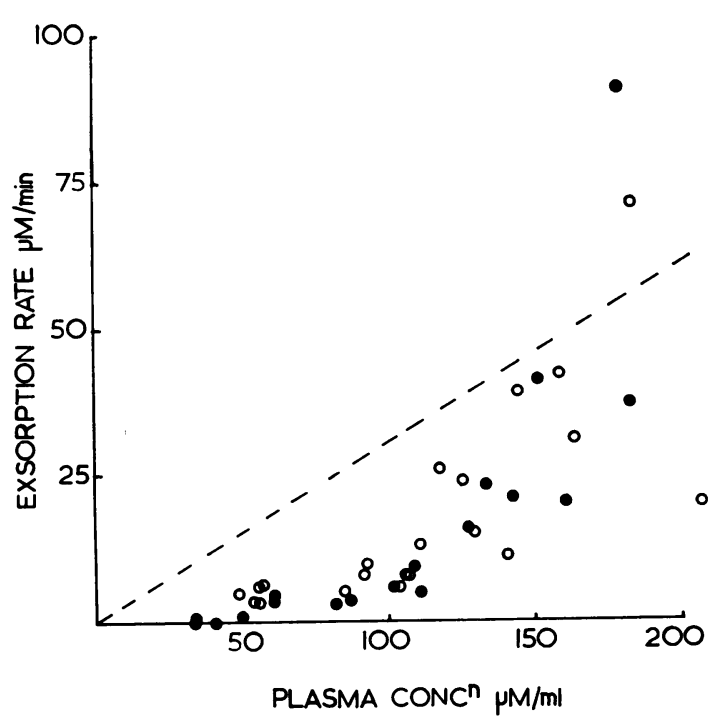

Fig 5 Galactose exsorption. The closed circles represent exsorption when the perfusate comprised physiological saline; the open circles show the effect of removing $\mathrm{Na}^{+}$from the perfusate.

unknown. They may pass between the enterocytes by diffusing through the junctional complexes or they may perhaps leak out at the tips of villi through the extrusion zones left by exfoliating cells. Alternatively they may gain access to the lumen by diffusing

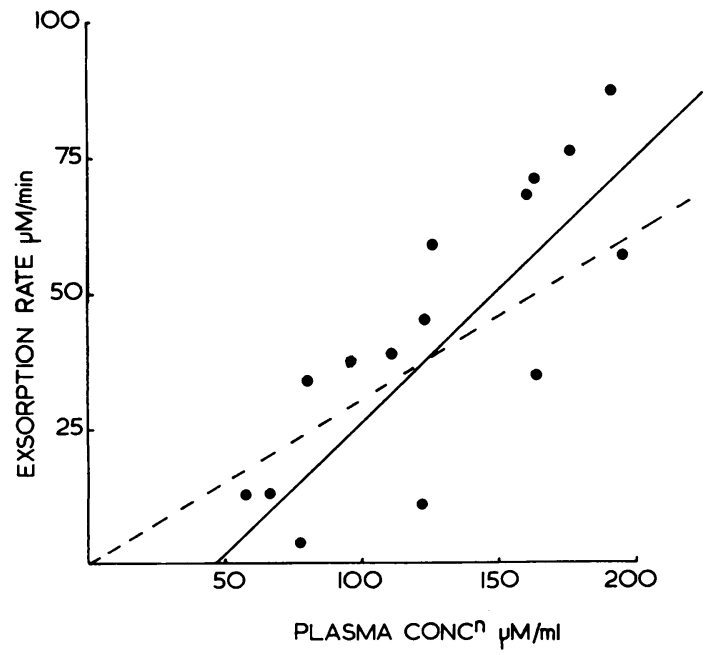

Fig 6 The exsorption pattern of glucose when the perfusate contained phlorizin $10^{-3} \mathrm{M}$.

The regression line is the continuous line $(y=0 \cdot 28 x$ $-13 \cdot 21, r=0 \cdot 822)$. The broken line represents an intestinal clearance of $0.3 \mathrm{ml} / \mathrm{min}$. 


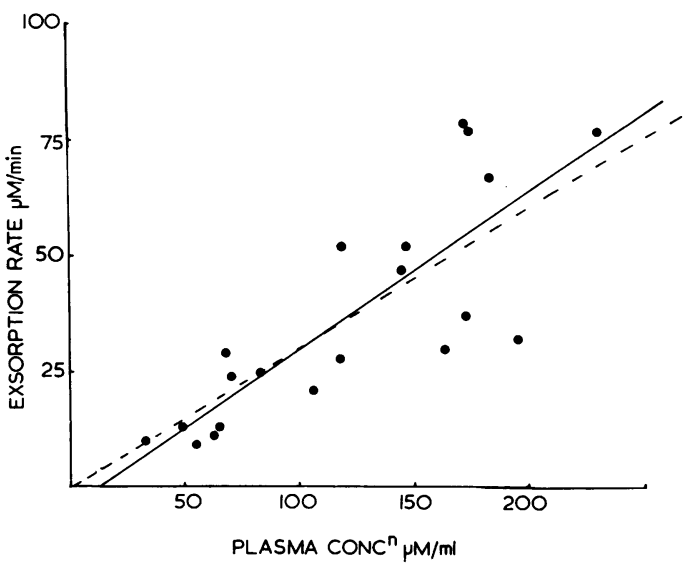

Fig 7 The exsorption pattern of glucose when the saline perfusate contained phlorizin $10^{-2} M(y=$ $0 \cdot 34 x-3 \cdot 78, r=0 \cdot 820)$.

directly through the enterocytes. The tight junction seems an unlikely route for egress because microscopically it comprises a $150 \mathrm{~nm}$ depth of fused cell membrane; however, there is evidence to suggest that some molecules, particularly ions, do use an extracellular route in passing between plasma and intestinal lumen (Schultz and Frizzell, 1972) and it must be admitted that the whole question of how tight is a tight junction remains unsolved. It is difficult to evaluate the importance of extrusion zones as possible areas of leakage.

The transcellular route implies that several barriers must be negotiated before an exsorbing molecule can escape into the lumen. These barriers comprise capillary wall, lamina propria, basement membrane, enterocyte cytoplasma, microvillus membrane, and fuzzy coat. This is the route which insorbing molecules are thought to take and it seems reasonable to suppose that exsorbing molecules will follow the same pathway in reverse.

The intestinal wall represents an anatomical obstruction to the passage of molecules, such that smaller ones are able to exsorb more quickly than large ones. Xylose exsorbs more rapidly than the two larger molecules, lactulose and mannose. The mechanism of exsorption in each of these cases is probably simple diffusion. This will account for the finding that exsorption is proportional to plasma concentration and that their exsorption rates approximate with those predicted from their molecular weights (Loehry et al, 1970).

Glucose and galactose have an exsorption pattern which is quite different from the three other sugars. This suggests that they exsorb by a separate route or that they are interfered with during their passage

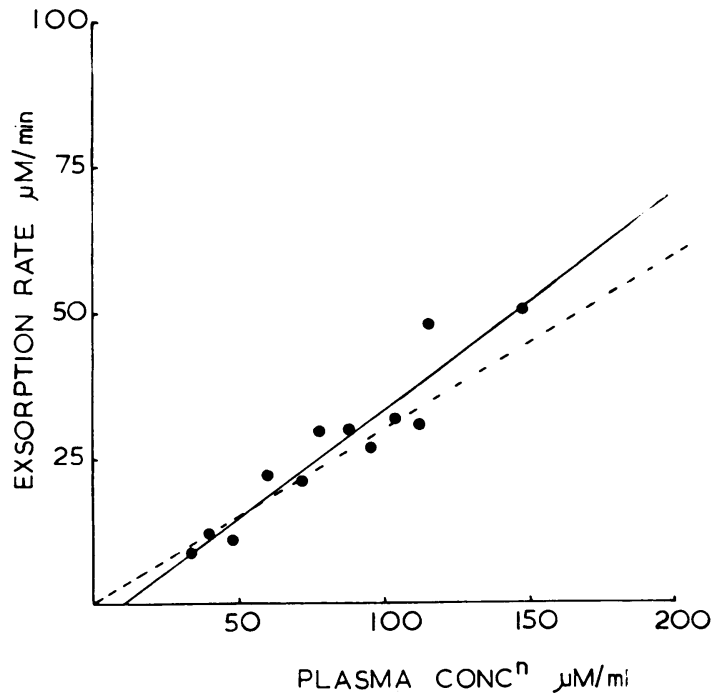

Fig 8 The exsorption of pattern galactose when the intestinal perfusate comprised equal parts phvsiological saline and isotonic glucose $(y=0.37 x-3 \cdot 39, r=0.917$.

across the intestinal wall. Lactulose is a disaccharide which contains a galactose substitute, so it would appear that the attachment of another monosaccharide to galactose takes away its characteristic exsorption properties. Xylose is thought to have an affinity for the sodium-sensitive glucose absorption mechanism (Csáky and Lassen, 1964); it is a little surprising therefore that it does not behave in the same way as glucose and galactose. The explanation for this may be that the exsorption pattern shown by glucose and galactose is not related to the sodiumsensitive absorption mechanism but to some other property they have in common; alternatively, it may be that the affinity of xylose for the absorption mechanism is very low.

Newey, Sanford, and Smyth (1963), studying glucose and maltose transfer in inverted sacs of intestine, showed that both sugars can enter the intestinal cell from the serosal fluid. Maltose passes from the serosal fluid through the intestinal wall, is hydrolysed at the mucosal edge of the cell, and the resulting glucose is normally reabsorbed into the cell. When phlorizin is present in the mucosal fluid the glucose liberated by maltase leaks out into the mucosal fluid.

Work on glucose exsorption in vivo has been done by Atkinson, Parsons, and Smyth (1957); they showed that at physiological plasma concentrations glucose did not pass into the intestinal lumen. Christensen and Gray (1963), however, showed that the non-metabolizable but actively transported 
hexose 3-0-me glucose did exsorb and that the presence of glucose in the luminal fluid speeded it up. They concluded that the exsorbing sugar was using the special glucose absorption mechanism. Cocco and Hendrix (1965) used high concentrations of plasma glucose $(50-70 \mu \mathrm{M} / \mathrm{ml})$; they showed that glucose exsorption in rats was very slow and that it was uninfluenced by luminal phlorizin $2 \times 10^{-3} \mathrm{M}$. They felt that a non-specific anatomical barrier, unrelated to the glucose absorption mechanism, prevented glucose exsorption.

The results presented here differ from those of previous authors because a wider range of plasma sugar concentrations was studied $(0-200 \mu \mathrm{M} / \mathrm{ml})$. The concentrations used by Cocco and Hendrix (1965) and Atkinson et al (1957) fall on the early part of the glucose exsorption curve where exsorption is very slow. It is more difficult to account for why Cocco and Hendrix were unable to show an increase in exsorption using phlorizin; higher concentrations of phlorizin were used in these experiments, or it may be that rabbit is more susceptible to this inhibitor than rat.

The results presented here show that in addition to an anatomical mucosal barrier which slows the passage of all large water-soluble molecules, there is a specific barrier which prevents the exsorption of glucose and galactose. Both glucose and galactose have a high affinity for the sodium-sensitive glucose absorption mechanism so it is probable that this property is responsible for the way in which they exsorb. Two features must be explained: first what is the barrier which prevents exsorption at plasma concentrations below $100 \mu \mathrm{M} / \mathrm{ml}$, and secondly why does the barrier fail when the plasma concentration is raised above this?

Figures 7 and 8 show that competitive inhibitors for the glucose absorption mechanism abolish the mucosal barrier when they are present in the intestinal lumen in high concentration. This suggests that the absorption mechanism is involved in maintaining the barrier, but it must be stressed that the concentration of phlorizin used in these experiments was very high, and that at these concentrations in vitro phlorizin has metabolic effects in addition to those of a competitive inhibitor.

2:4 Dinitrophenol (DNP) is an inhibitor of aerobic respiration. Detheridge, Matthews, and Smyth (1966) showed in vitro that in a concentration of $2 \times 10^{-4} \mathrm{M}$ it prevented active glucose absorption. The glucose/galactose absorption mechanism depends on metabolic energy to absorb these sugars against a concentration gradient. Two possible explanations are available to account for the failure of DNP to break down the barrier: either it is not effective in suppressing metabolismat the concentration used, or alternatively, the barrier is not maintained by metabolism. The first explanation is probably the correct one, but the possibility remains that there might be one mechanism which specifically insorbs glucose and is dependent upon energy while a second one, possibly independent of energy, prevents the sugar from flowing back into the lumen.

Figure 5 shows that the intestine will maintain a transmucosal concentration difference of galactose in the absence of luminal sodium; Axon and Creamer (1971) have shown that the same is true for glucose. It has been widely assumed that these sugars cannot be absorbed against a concentration gradient unless luminal sodium is present. The most likely explanation for this finding is that there is a sodium-rich micro-environment bounded by the fuzzy coat externally and the microvillus membrane internally. This would apply if sodium continuously exsorbed from the plasma while the fuzzy coat acted as a diffusion barrier (Hamilton and McMichael, 1968).

The barrier to glucose exsorption can be explained in two ways: either glucose reaches the lumen and is actively reabsorbed again, or, alternatively, the active mechanism intercepts glucose before it reaches the lumen and turns it back. It is uncertain which of these possibilities is correct, but it seems reasonable to assume that the barrier to glucose and galactose exsorption is related to the special transport mechanism.

The barrier is completely effective at a plasma level of $50 \mu \mathrm{M} / \mathrm{ml}$ but after this it begins to fail, and, as the plasma concentration rises, the barrier disappears so that at concentrations above 150 $\mu \mathrm{M} / \mathrm{ml}$, glucose exsorption is greater than $0.3 \mathrm{ml} /$ min, ie, greater than that which would be expected calculating from its molecular weight. It is difficult to account for the failure of the barrier on the basis of a simple one-way glucose pump. If a one-way pump was restricting the passage of glucose and it was stressed by an increasing glucose load, it would begin to leak when its maximum reabsorptive capacity was reached, but no matter what the glucose load, it would continue to pump glucose back and glucose exsorption would never reach that of mannose.

Many workers use a carrier model to explain glucose absorption, and certain modifications have been proposed to account for the observation that glucose may be transported into the cell against a concentration gradient (Schultz and Curran, 1970; Crane, 1968). It is clear that a bidirectional mechanism can transport sugar from plasma to lumen as well as from lumen to plasma. Under normal circumstances it works from lumen to plasma with a built-in affinity bias producing an intracellular accumulation of sugar, but if stressed sufficiently by 
increasing the plasma sugar concentration it might be forced to work backwards. This may account for the findings presented here, but one other possible explanation could be that at high plasma concentrations the enterocytes are injured either by the high concentration of sugar in the plasma or from osmotic changes. This seems unlikely because no damage was detected on light microscopy and in any case, this explanation would not account for the way that glucose exsorption exceeded $0.3 \mathrm{ml} / \mathrm{min}$ at very high plasma concentrations.

The data presented in this paper suggest that there are two barriers which prevent actively absorbed monosaccharides from passing from plasma to lumen: an anatomical one which restricts all large water-soluble molecules to some extent, and the other a specific barrier which is related to the active absorption mechanism. At high plasma concentrations the specific barrier to glucose breaks down; it may be that a bidirectional element in the glucose absorption mechanism facilitates glucose exsorption when plasma glucose is very high.

We wish to thank Miss J. Cole for technical assistance with the experimental work and $\mathrm{Mr} \mathrm{D}$. Altman for statistical help. We are grateful to St Thomas' Hospital Endowment Committee for a generous financial grant which enabled A.T.R.A. to spend a year doing gastrointestinal and experimental work. Part of this work contributed to an M.D. thesis, accepted by the University of London, 1973.

Requests for reprints should be addressed to
A.T.R.A., Gastrointestinal Laboratory, St Thomas' Hospital, London SE1.

References

Atkinson, R. M., Parsons, B. J., and Smyth, D. H. (1957). The intestinal absorption of glucose. J. Physiol. (Lond.), 135, 581589.

Axon, A. T. R., and Creamer, B. (1971). Glucose exsorption stimulated by a competitive inhibitor. J. Physiol. (Lond.), 215, 6-7P.

Christensen, H. N., and Gray, S. J. (1963). Reversibility of intestinal absorption of 3-0-methyl glucose. Proc. Soc. exp. Biol. (N.Y.), 114, 215-217.

Cocco, A. E., and Hendrix, T. R. (1965). The effect produced by phlorizin and hypertonic saline on sugar movement from blood to intestinal lumen. Bull. Johns Hopk. Hosp., 117, 296-305.

Code, C. F. (1960). The semantics of the process of absorption. Perspect. Biol. Med., 3, 561-562.

Csáky, T. Z., and Lassen, U. V. (1964). Active intestinal transport of D-xylose. Biochim. biophys. Acta (Amst.), 82, 215-217.

Crane, R. K. (1968). Absorption of sugars. In Handbook of Physiology, Sect. 6, Alimentary Canal, edited by C. F. Code, vol. III, pp. 1323-1351. American Physiological Society, Washington, D.C.

Detheridge, J. F., Matthews, J., and Smyth, D. H. (1966). The effect of inhibitors on intestinal transfer of glucose and fluid. $J$. Physiol. (Lond.), 183, 369-377.

Hamilton, J. D., and McMichael, H. B. (1968). Role of the microvillus in the absorption of disaccharides. Lancet, 2, 154-157.

Loehry, C. A., Axon, A. T. R., Hilton, P. J., Hider, R. C., and Creamer, B. (1970). Permeability of the small intestine to substances of different molecular weight. Gut, 11, 466-470.

Menzies, I. S. (1973). Quantitative estimation of sugars in blood and urine by paper chromatography using direct densitometry. J. Chromatogr., 81, 109-127.

Newey, H., Sanford, P. A., and Smyth, D. H. (1963). Location of function in the intestinal epithelial cell in relation to carbohydrate absorption. J. Physiol. (Lond.), 168, 423-434.

Schultz, S. G., and Curran, P. F. (1970). Coupled transport of sodium and organic solutes. Physiol. Rev., 50, 637-718.

Schultz, S. G., and Frizzell, R. A. (1972). An overview of intestinal absorptive and secretory processes. Gastroenterology, 63 161-170. 